\title{
An initial investigation of the long-term trends in the fluxgate magnetometer (FGM) calibration parameters on the four Cluster spacecraft
}

\author{
L. N. S. Alconcel, P. Fox, P. Brown, T. M. Oddy, E. L. Lucek, and C. M. Carr \\ Department of Physics, The Blackett Laboratory, Imperial College London, Prince Consort Road, London, SW7 2BW, UK \\ Correspondence to: L. N. S. Alconcel (1.alconcel@imperial.ac.uk)
}

Received: 25 October 2013 - Published in Geosci. Instrum. Method. Data Syst. Discuss.: 27 January 2014

Revised: 29 May 2014 - Accepted: 6 June 2014 - Published: 11 July 2014

\begin{abstract}
Over the course of more than 10 years in operation, the calibration parameters of the outboard fluxgate magnetometer (FGM) sensors on the four Cluster spacecraft are shown to be remarkably stable. The parameters are refined on the ground during the rigorous FGM calibration process performed for the Cluster Active Archive (CAA). Fluctuations in some parameters show some correlation with trends in the sensor temperature (orbit position). The parameters, particularly the offsets, of the spacecraft 1 (C1) sensor have undergone more long-term drift than those of the other spacecraft (C2, C3 and C4) sensors. Some potentially anomalous calibration parameters have been identified and will require further investigation in future. However, the observed longterm stability demonstrated in this initial study gives confidence in the accuracy of the Cluster magnetic field data. For the most sensitive ranges of the FGM instrument, the offset drift is typically $0.2 \mathrm{nT}$ per year in each sensor on $\mathrm{C} 1$ and negligible on $\mathrm{C} 2, \mathrm{C} 3$ and $\mathrm{C} 4$.
\end{abstract}

\section{Introduction}

The Cluster mission (Escoubet et al., 1997) consists of four Earth-orbiting spacecraft flying in formation at variable separations $(100-10000 \mathrm{~km})$. The science phase of the mission began in February of 2001 and is presently scheduled to continue until December 2016 (pending final confirmation by the European Space Agency). Mission scientists study smallscale plasma structures in space and time in key regions of the magnetosphere, including the solar wind, the bow shock, the magnetopause, the polar cusps, the magnetotail and the auroral zones (Walsh et al., 2010). Each spacecraft carries the same set of eleven instruments which detect spatial and temporal changes in the magnetosphere by measuring ambient electromagnetic fields and particle populations. FGM is a DC (direct current) magnetometer used to measure the magnetic field vector at the instrument's position (Balogh et al., 1997).

Each FGM instrument consists of two triaxial fluxgate sensors. They are boom-mounted to minimise interference from the spacecraft's background magnetic field, and the outboard sensor at the end of the $5 \mathrm{~m}$ boom is designated as the primary sensor for science data. The sensors can be operated in several ranges depending on the spacecraft's location in the magnetosphere, covering magnetic field magnitudes from less than $1 \mathrm{nT}$ to over $65000 \mathrm{nT}$ (see Table 1). Data are normally obtained at a rate of $\sim 22$ vectors per second $(\mathrm{Hz})$, designated as "normal mode", although this can be increased to $\sim 67 \mathrm{~Hz}$ for short periods to investigate a region or event of particular interest ("burst mode").

After the raw data are downlinked, they are processed into a usable format and the time at which the data were measured is reconstructed. They are subsequently calibrated, validated and processed into the final FGM data products which appear on the Cluster Active Archive (CAA) (Laakso et al., 2010). Submission to the CAA occurs once all of these procedures have been performed on 1 month's worth of data, which is divided into orbits, defined as the periods between successive periapses. Orbit period varies from 51 to $57 \mathrm{~h}$ depending on the phase of the mission, with the orbits shortening as the mission progresses.

The four Cluster spacecraft are magnetically very clean, giving a high level of confidence in the DC magnetic field 
Table 1. FGM instrument ranges.

\begin{tabular}{cl}
\hline Range number & B \\
\hline 2 & -64 to $63.97 \mathrm{nT}$ \\
3 & -256 to $255.87 \mathrm{nT}$ \\
4 & -1024 to $1023.5 \mathrm{nT}$ \\
5 & -4096 to $4094 \mathrm{nT}$ \\
6 & -16384 to $16376 \mathrm{nT}$ \\
7 & -65536 to $65504 \mathrm{nT}$ \\
\hline
\end{tabular}

data obtained by the FGM instruments. The combination of measurement and modelling on the ground with a rigorous magnetic cleanliness programme and final compensation for magnetic contributions means that the spacecraft field at the outboard magnetometer sensors should be less than $0.25 \mathrm{nT}$ (Balogh et al., 1997). It is not possible to verify this in-flight, however.

The accurate calibration of the FGM instrument is critical for scientific investigations requiring high-accuracy vector magnetic field data, for the production of some data sets by other instruments (PEACE, RAPID) and for the calibration of other instruments aboard the Cluster spacecraft (EFW, STAFF, WHISPER).

\section{FGM calibration}

In order to place the parameter trends into context, it is useful to describe briefly the calibration, validation and archiving procedures. These are described in detail elsewhere (Gloag et al., 2010).

\subsection{Theory}

The FGM magnetic field data are subject to several significant sources of error that must be corrected to yield the best results for use in scientific studies and for use by other Cluster instruments. In the coordinate transformation of the magnetic field data from the sensor measurement frame to the spacecraft reference frame (and hence to a geophysical frame), errors may arise due to incomplete knowledge of (i) the orientation of the sensors' axes, (ii) the sensor offsets, and (iii) the sensor gains.

The relationship between the measurement frame and the spacecraft reference frame is specified by a set of 12 parameters for each spacecraft, as shown in Eq. (1) below. The set consists of the sensor angles, gains and offsets. The calibration parameters then define a transformation of the following form:

$$
\left(\begin{array}{l}
B_{S_{1}} \\
B_{S_{2}} \\
B_{S_{3}}
\end{array}\right)=\left(\begin{array}{lll}
G_{1} \sin \vartheta_{1} \cos \phi_{1} & G_{1} \sin \vartheta_{1} \sin \phi_{1} & G_{1} \cos \vartheta_{1} \\
G_{2} \sin \vartheta_{2} \cos \phi_{2} & G_{2} \sin \vartheta_{2} \sin \phi_{2} & G_{2} \cos \vartheta_{2} \\
G_{3} \sin \vartheta_{3} \cos \phi_{3} & G_{3} \sin \vartheta_{3} \sin \phi_{3} & G_{3} \cos \vartheta_{3}
\end{array}\right)
$$

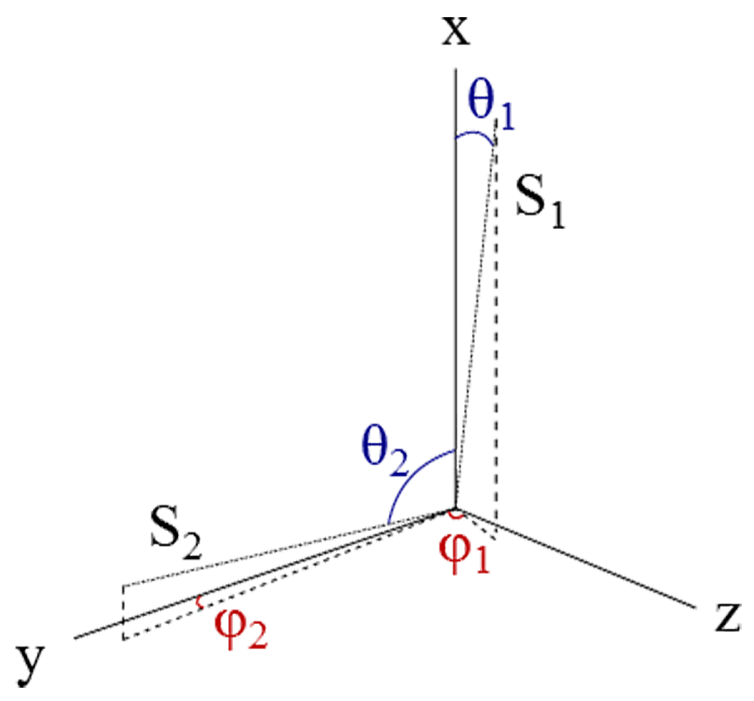

Figure 1. The relation between the orthogonal $(x, y, z)$ and sensor $\left(S_{1}, S_{2}, S_{3}\right)$ coordinate systems. The elevation and azimuthal angles $\theta$ and $\varphi$ for each sensor coordinate are defined in the same way. $S_{3}$ has been omitted for clarity.

$$
\left(\begin{array}{c}
B_{x} \\
B_{y} \\
B_{z}
\end{array}\right)+\left(\begin{array}{c}
O_{1} \\
O_{2} \\
O_{3}
\end{array}\right)
$$

$\left(B_{x}, B_{y}, B_{z}\right)$ is the magnetic field vector in the spinning spacecraft coordinate system, where $x$ is aligned along the spin axis of the spacecraft and $y, z$ are located in the spin plane, forming an orthogonal triad. In this equation, $\left(B_{S 1}\right.$, $\left.B_{S 2}, B_{S 3}\right)$ represents the magnetic field vector as measured in the non-orthogonal sensor coordinate system, where $S_{1}$, $S_{2}$ and $S_{3}$ point approximately along the spacecraft $x, y$ and $z$ axes respectively. The parameters describing the transformation are the offsets $\left(O_{i}\right)$, gains $\left(G_{i}\right)$, elevation angles $\left(\theta_{i}\right)$ and azimuthal angles $\left(\varphi_{i}\right)$. The elevation angle is measured with respect to the spacecraft spin axis $x$; the azimuthal angle is measured around from the $y$ axis in the spin plane $y-z$. Figure 1 illustrates the relationship between two reference frames. The gains and angles in the coupling matrix orthogonalise, scale and orient the field measured by the sensors, while the offsets handle zeroing the sensors.

These calibration parameters were accurately measured on the ground at the Technical University of Braunschweig as part of the pre-flight calibration of FGM. However, these parameters cannot be expected to remain constant over the time scale of the mission; thus, in order to maintain the quality of the measured magnetic field data, an in-flight calibration process is required. As a mission consisting of multiple spinning spacecraft which spend significant portions of their time in the solar wind, Cluster represents an opportunity to bring several magnetometer calibration methods to bear. 
The in-flight calibration technique is based upon two distinct methods: a Fourier analysis method (Kepko et al., 1996), which recovers 8 of the 12 calibration parameters, and a solar wind analysis method, which recovers the spin-axis offsets $O_{1}$ (Hedgecock, 1975). A brief description of the theory underlying these methods, together with a discussion of their limitations and constraints on their application, is given below. Note that in addition to being orthogonalised and transformed into spacecraft coordinates, the magnetic field components must also be despun. For the sake of brevity, the despinning procedure will not be outlined here.

\subsection{Fourier analysis}

The Fourier analysis is based on the procedure detailed in Kepko et al. (1996). When the magnetic field data are despun, errors in particular calibration parameters will produce coherent monochromatic signals at the first and second harmonics of the spin frequency (approximately 0.25 and $0.5 \mathrm{~Hz}$ for the Cluster spacecraft). More specifically: errors in the spin-plane elevation angles $\left(\theta_{2}, \theta_{3}\right)$ and spin-plane offsets $\left(\mathrm{O}_{2}, \mathrm{O}_{3}\right)$ produce signals at the first harmonic in the spinplane components of the field; errors in the relative spinplane azimuthal angles $\left(\Delta \varphi_{32}\right)$ and relative spin-plane gains $\left(\Delta G_{32}\right)$ produce signals at the second harmonic in the spinplane components of the field; and errors in the spin-axis elevation angle $\theta_{1}$ and spin-axis azimuthal angle $\varphi_{1}$ produce signals at the first harmonic in the spin-axis component of the field.

Fourier-transforming the despun data produces a set of equations containing the errors in the above calibration parameters, which can then be inverted to recover the values of those parameters. The errors in the remaining four parameters $\left(G_{1}, O_{1}, G_{3}, \varphi_{3}\right)$ do not produce coherent signals in the despun data and so they cannot be recovered by this method. After the Fourier analysis, the residual signal power at the first and second harmonics of the spin frequency provides one of the measures by which the accuracy of the calibration can be judged.

\subsection{Solar wind analysis}

In general, the four Cluster spacecraft sample the solar wind from mid-December to mid-April, a period which is known as the "dayside" season. During this period, the magnetic field in the solar wind is used to adjust the offset $\left(O_{1}\right)$ associated with the axis of the sensor that is aligned with the spin axis of the spacecraft. FGM is nearly always in range 2 during these periods, so this is the only range for which this method can be used to refine the spin-axis offset. This procedure is based on the observation that fluctuations in the solar wind magnetic field are primarily rotational, which means that there should be no correlation between the spin-axis component of the magnetic field and the total field magnitude (Hedgecock, 1975).
The procedure works by searching through the spinaveraged data for rotational discontinuities. At these discontinuities, $O_{1}$ is adjusted to minimise the correlation between $B_{1}$ and $|B|$. In general, 1 month's worth of data is divided in half and adjustments are applied separately to the first and second halves of the month. The implementation of this procedure was originally developed by FGM co-investigators at UCLA (University of California Los Angeles; personal communication with H. K. Schwarzl, K. Khurana, and M. Kivelson, 2005) who have collaborated with the FGM team on its implementation at Imperial College. A complete description of the theory underlying this method can be found in Hedgecock (1975).

From mid-April to mid-December the four Cluster spacecraft sample Earth's magnetotail, a period which is known as the "nightside" or "tail" season. The technique described above cannot be applied to this data to adjust the spin-axis offset. A simple linear interpolation of the offset between the last solar wind measurement in mid-April and the first solar wind measurement in mid-December is performed instead. This method likely masks the natural variation in the offset during these periods.

\subsection{Range changes}

When the FGM switches between ranges (Table 1), the magnetic field components are not precisely equal on either side of the change, due to differences in calibration between different ranges. In order to mitigate this, adjustments are performed to the remaining parameters not determined by either of the above procedures; namely $O_{1}$ (ranges 3 and above), $G_{1}, G_{3}$ and $\varphi_{3}$. These parameters are adjusted from their measured values on the ground in order to minimise the discontinuities in the field components that occur at the range change. In common with the solar wind analysis, the implementation of this procedure was originally developed by FGM co-investigators at UCLA (personal communication with H. K. Schwarzl, K. Khurana, and M. Kivelson, 2005) who have also collaborated with the FGM team on its implementation at Imperial College.

\subsection{Validation and archiving}

Once the calibration procedure has been completed, visual inspection of the calibrated data is carried out as a qualitycontrol step. The accuracy of the calibration parameters recovered by the Fourier analysis method manifests itself in the signal power at the spin frequency in the processed data. The accuracy of the spin-axis offset recovered by the solar wind method manifests itself in the spread exhibited between the four spacecraft's spin-axis data in the solar wind. The limitations of the calibration procedures mean that the quality of the final calibration can vary from month to month. Data intervals which do not meet the minimum standard for calibration quality are flagged in caveat files which accompany 


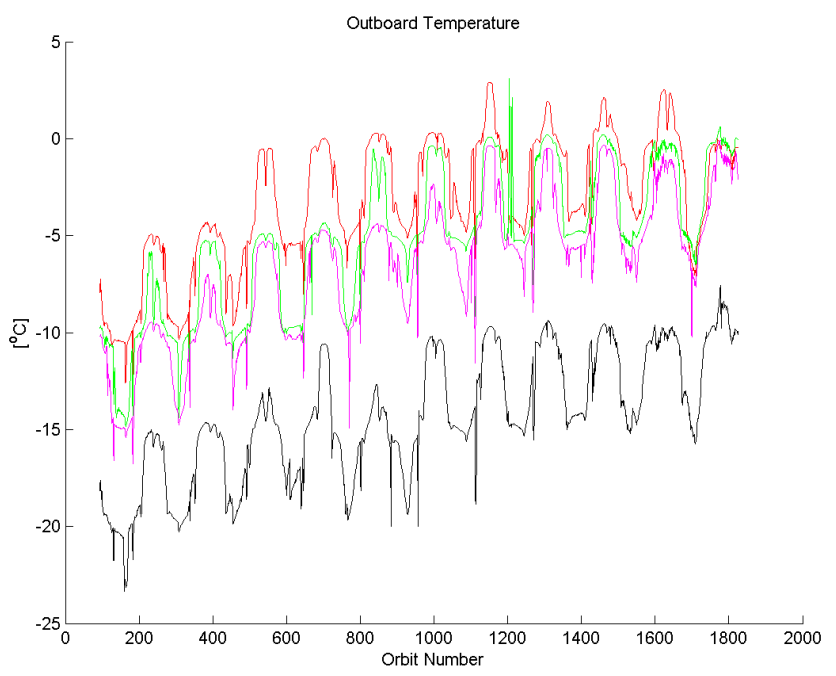

Figure 2. Outboard sensor temperatures in degrees Celsius for each spacecraft for orbits 93-1889 (February 2001-August 2012). C1 black, C2 - red, C3 - green, C4 - magenta.

the FGM data products on the CAA. Additionally, a calibration file for each orbit is produced. They are made available to investigators on the CAA; however, since the FGM data products are already calibrated, they simply list the calibration parameters for each range in the orbit. The CAA web site (http://caa.estec.esa.int/caa/) gives researchers access to the data from all of the instruments on board Cluster from the start of the mission. Documentation and software tools are also downloadable.

\subsection{Application, limitations and uncertainties of the calibration procedures}

Having presented a brief account of the theory underlying the FGM calibration procedure above, it is also worthwhile to discuss some of the practicalities involved in applying these procedures to the FGM data, together with limitations of the calibration procedures and quantification of remaining uncertainty in the calibration parameters.

The parameters recovered by the Fourier analysis method are resolved most frequently, in practice, once per orbit. The remaining parameters are determined less often. Accurate determination of the spin-axis offset by the solar wind method requires a minimum of $20 \mathrm{~h}$ of good quality solar wind data. Accordingly, during the dayside season, the spin-axis offset is typically only determined twice per month. The range jump correction is usually performed once per month.

There are two primary criteria used to determine the accuracy of the calibration results. The first is the spread in the spin-axis components of the magnetic field in the solar wind, as measured by each of the four spacecraft. Under quiet solar wind conditions, it is to be expected that the distance between the Cluster spacecraft is small compared to the dis-

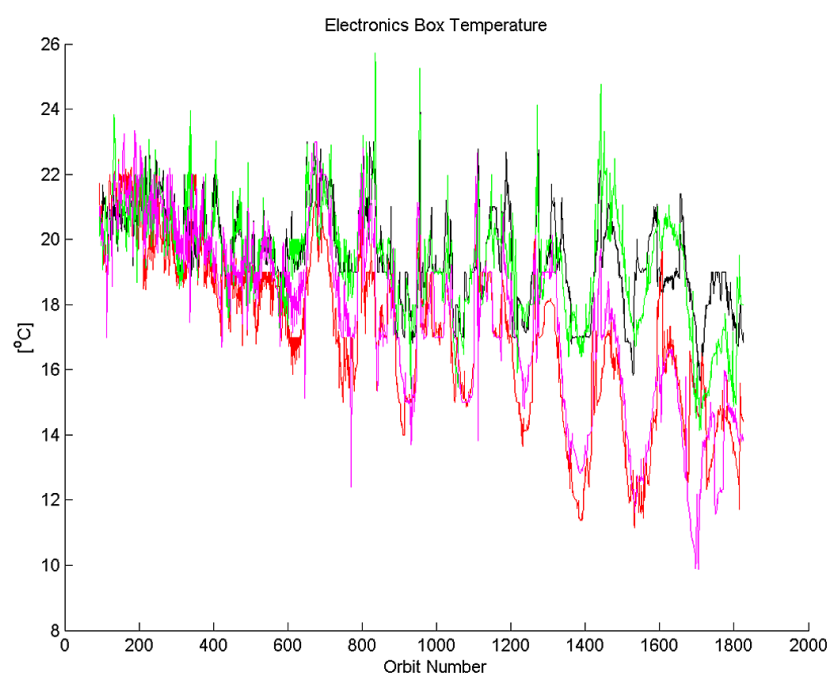

Figure 3. Electronics box temperatures in degrees Celsius for each spacecraft for orbits 93-1889 (February 2001-August 2012). C1 black, C2 - red, C3 - green, C4 - magenta.

tance scales over which the solar wind magnetic field varies significantly. Accordingly, a small spread in $B_{x}$ is the criterion by which the accuracy of the determined values of $O_{1}$ are measured.

The second criterion is the signal power in the spin-axis and spin-plane components of the magnetic field, measured at the first and second harmonics of the spin frequency. This quantity is used to measure the quality of the Fourier analysis component of the calibration procedure. It is frequently the case that the spin-axis parameters recovered by the Fourier analysis method have produced an inferior calibration to that obtainable by using calibration parameters from a previous orbit. Accordingly, in such cases, multiple spin-axis calibrations were substituted, and the set of parameters which produced the minimum spin power in the spin-axis data was chosen as the final calibration.

While these criteria provide an estimate of the quality of the calibration, they cannot be used to determine the remaining uncertainty in any individual calibration parameter. In particular, it should be noted that the set of calibration parameters determined by the Fourier analysis method is solely chosen to minimise the signal power at the first and second harmonics of the spin frequency. There may be other sets of values for these parameters which would satisfy this criterion equally well. Accordingly, lacking an independent reference for the individual field components, caution must be used when discussing how these calibration parameters relate to the true gains and alignment angles of the instrument. For example, within a time period as short as an orbit, it is reasonable to assume that the alignment angles of the instrument are independent of the operating range. However, the angles output by the Fourier analysis procedure do vary slightly between ranges, again because the software implementation 

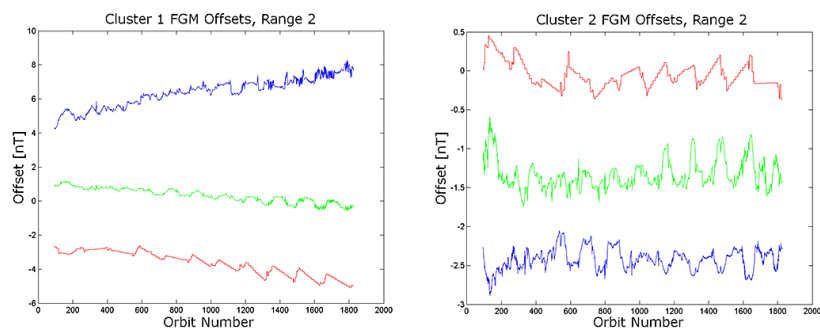

Cluster 3 FGM Offsets, Range 2
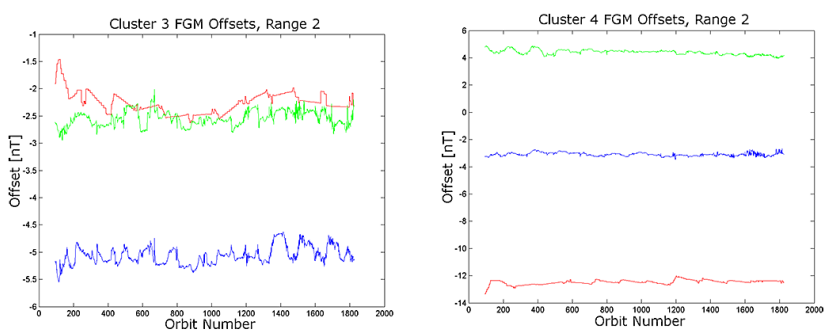

Figure 4. Range 2 spin-axis $\left(O_{1}\right.$, red $)$ and spin-plane $\left(O_{2}\right.$ and $O_{3}$, blue and green) offsets in nanoteslas for orbits 93-1825 (February 2001-February 2012).
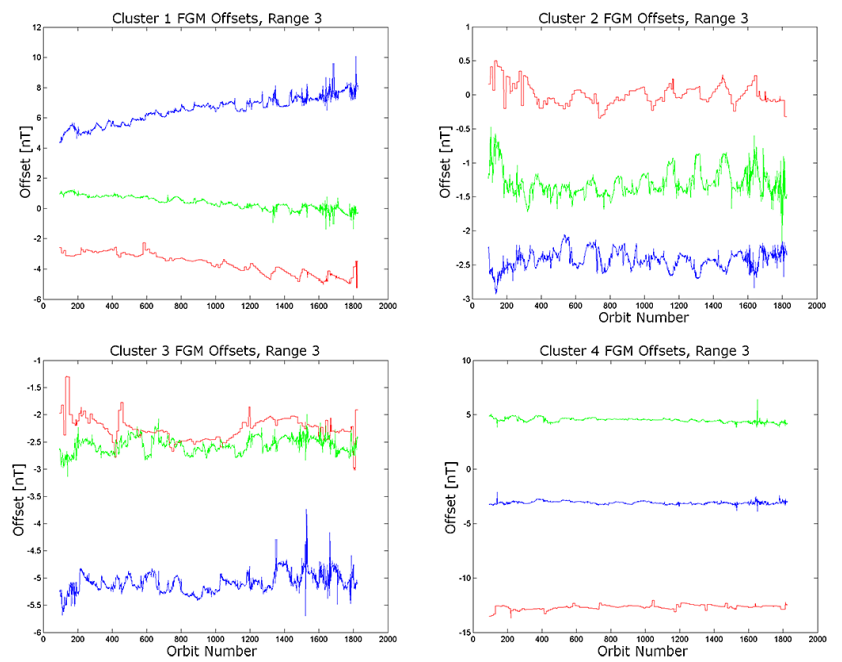

Figure 5. Range 3 spin-axis $\left(O_{1}\right.$, red $)$ and spin-plane $\left(O_{2}\right.$ and $O_{3}$, blue and green) offsets in nanoteslas for orbits 93-1825 (February 2001-February 2012).

only selects values which best reduce the spin-frequency signal power. No attempt is made to harmonise the alignment angles across different ranges. This discussion also underlines the importance of monitoring the output of the calibration process, as it is possible for unrealistic parameter values to be produced by the automated calibration routines.

The accuracy of the recovered parameters is strongly dependent upon the quality of the data available. Excessive signal noise, data gaps, etc. can all affect the efficacy of the calibration procedures. Periods of unavoidably poor calibration are flagged in FGM's CAA caveat files.
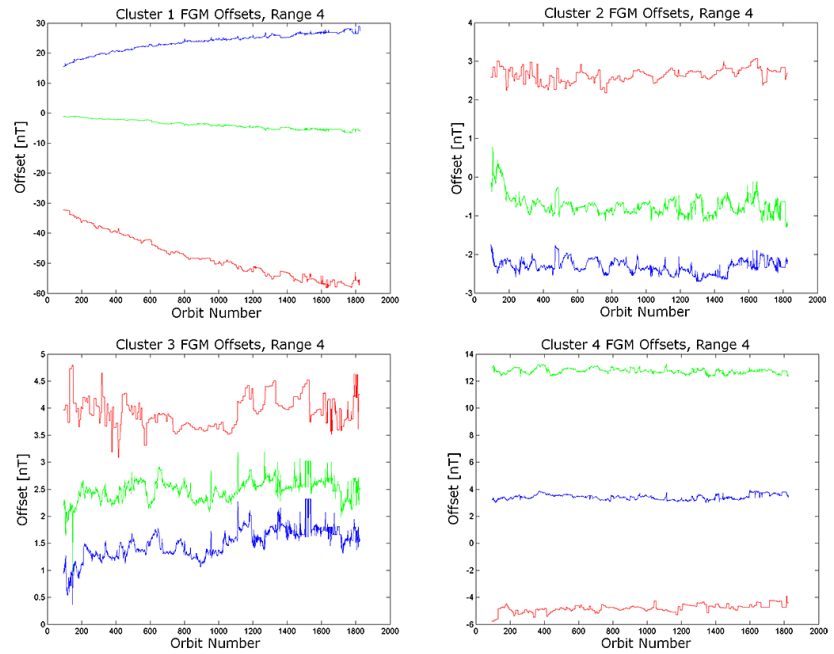

Figure 6. Range 4 spin-axis $\left(O_{1}\right.$, red) and spin-plane $\left(O_{2}\right.$ and $O_{3}$, blue and green) offsets in nanoteslas for orbits 93-1825 (February 2001-February 2012).

The original Cluster mission has been extended several times and utilised manoeuvres to configure a range of different spacecraft constellations. Trajectories bringing the spacecraft closer to Earth than originally foreseen necessitated the use of the full instrument ranging capability. From November 2000 to October 2006, ranges 2-4 (see Table 1) were in regular use. Starting in November 2006, range 5 entered routine use. Starting in May 2008, range 6 entered routine use. Starting in December 2009, range 7 entered routine use. Neither range 6 nor range 7 was originally intended for use during the nominal mission hence these ranges were not fully calibrated on the ground. The entry of the spacecraft into the inner magnetosphere and auroral acceleration zone in the extended mission phases meant that the total field magnitude exceeded the capacity of range 5 . The calibration parameters for range 6 and range 7 are tied to those of range 5, as only partial ground calibration information was available for them.

\section{Long-term trends in FGM parameters}

Having applied the above described calibration methods to the Cluster FGM data set over a period of 11 years, we considered it valuable to begin an examination of the long-term behaviour of the FGM calibration parameters. Such a survey serves several purposes:

- It allows us to examine the long-term measurement stability of the FGM instrument. Such stability has been observed in other space-based fluxgate magnetometers such as those aboard the CHAMP (Challenging Minisatellite Payload) and THEMIS (Thermal Emission 

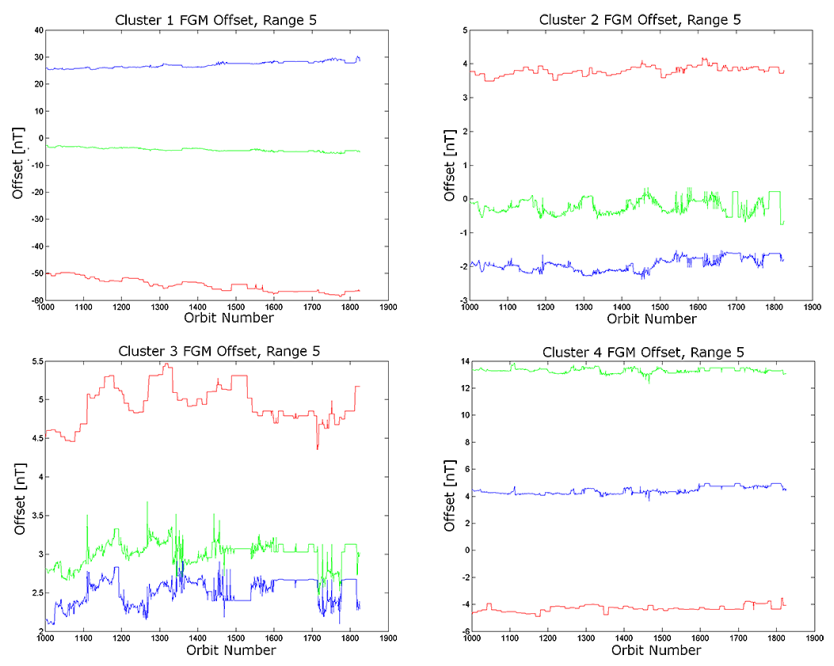

Figure 7. Range 5 spin-axis $\left(O_{1}\right.$, red $)$ and spin-plane $\left(O_{2}\right.$ and $O_{3}$, blue and green) offsets in nanoteslas for orbits 1000-1812 (December 2006-February 2012).

Imaging System) satellites (Auster et al., 2008; Yin and Luehr, 2011).

- It allows us to quickly identify periods where the calibration parameters have anomalous values, flagging data that may need to be revisited to see if the calibration can be improved. The most egregious cases have already been corrected as a result of the survey, and the data resubmitted to the CAA.

- It allows us to examine whether or not it is possible to correlate variations in calibration parameters with instrument and spacecraft events, particularly FGM instrument housekeeping telemetry.

- It gives some indication of the validity of interpolating the spin-axis offsets across the tail season, by comparing the change in offset over the tail seasons with overall variation throughout the mission.

More generally, a time history of FGM calibration on board the four Cluster spacecraft represents a unique and valuable body of knowledge in the field of space magnetometry, which should serve to inform the planning of any similar future missions where accurate magnetometer data are important. The 11 years of data discussed in this paper represent an opportunity to examine the results of a calibration campaign of unprecedented duration.

It should be noted that this paper aims to provide a primarily descriptive account of the results of the Cluster FGM calibration campaign over the period 2001-2012. No attempt has been made to quantify the remaining uncertainty in the FGM parameters after calibration, nor to incorporate these results into a comprehensive error analysis of the FGM instrument. Such an analysis lies outside the scope of this paper.
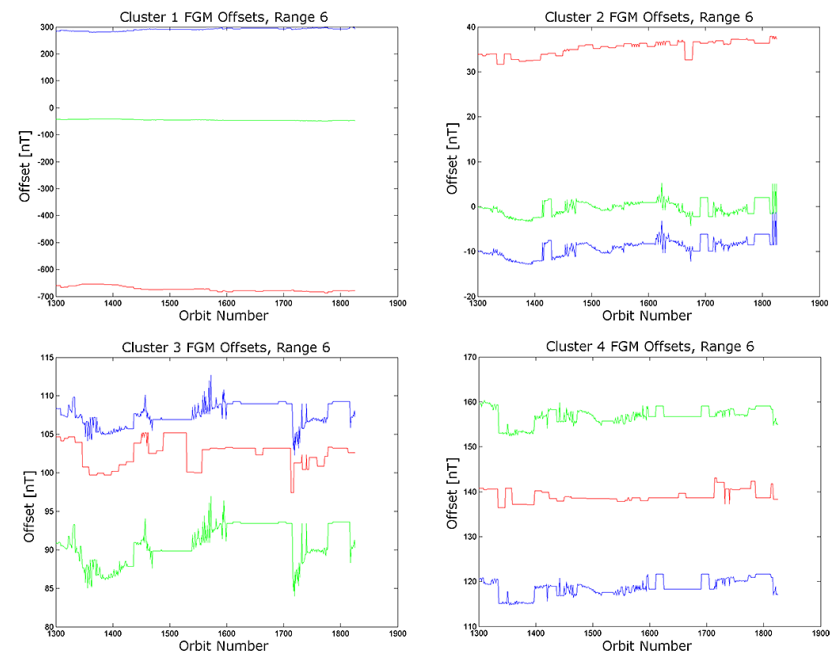

Figure 8. Range 6 spin-axis $\left(O_{1}\right.$, red) and spin-plane $\left(O_{2}\right.$ and $O_{3}$, blue and green) offsets in nanoteslas for orbits 1300-1825 (December 2008-February 2012).
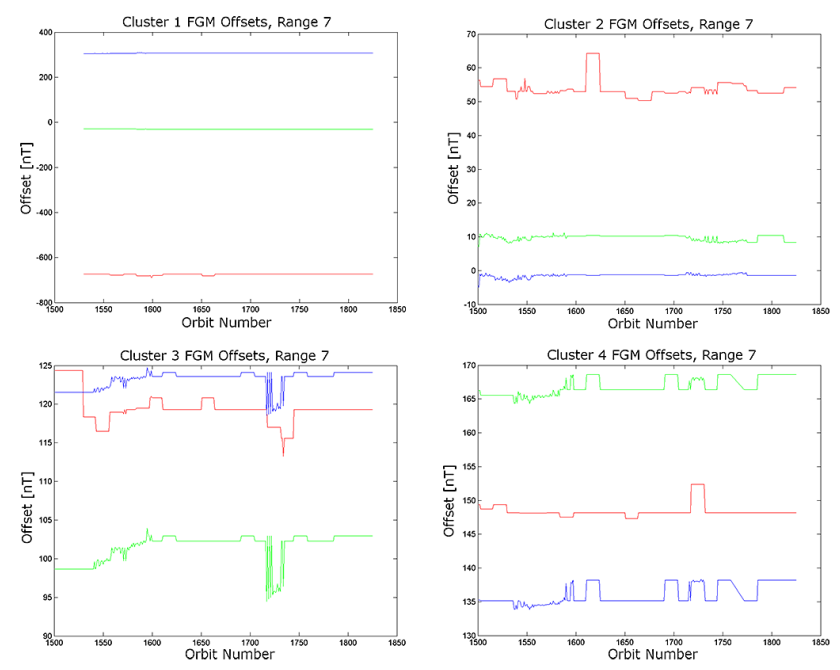

Figure 9. Range 7 spin-axis $\left(O_{1}\right.$, red) and spin-plane $\left(O_{2}\right.$ and $O_{3}$, blue and green) offsets in nanoteslas for orbits 1445-1825 (December 2009-February 2012).

The remainder of this paper consists of several parts. The entire data set consists of the time series for each calibration parameter, covering the period from the start of the nominal mission at orbit 93 (February 2001) to orbit 1825 (February 2012). Presentation and discussion of the time series for each parameter is impractical, given that the complete data set encompasses 12 parameters for each of the 6 ranges for each of the 4 Cluster spacecraft. Therefore, only a representative subset of the calibration parameters is discussed, highlighting what we consider the most significant features of the data set. 
Table 2. Mean and standard deviation of the offsets $\left(O_{i}\right)$ for the mission segment February 2001 to February 2012 for each coordinate in every range on all spacecraft. Standard deviations for ranges that are at least twice as large as those for all the other spacecraft in that range are highlighted in italic.

\begin{tabular}{|c|c|c|c|c|c|c|c|c|c|c|c|c|c|}
\hline \multicolumn{14}{|c|}{ Offset (nT) } \\
\hline \multicolumn{7}{|c|}{ Mean } & \multicolumn{7}{|c|}{ Standard deviation } \\
\hline \multicolumn{7}{|c|}{ Coordinate 1 (spin axis) } & \multicolumn{7}{|c|}{ Coordinate 1 (spin axis) } \\
\hline & Range 2 & Range 3 & Range 4 & Range 5 & Range 6 & Range 7 & & Range 2 & Range 3 & Range 4 & Range 5 & Range 6 & Range 7 \\
\hline Cluster 1 & -3.6472 & -3.6363 & -47.7804 & -54.3680 & -673.5475 & -674.3962 & Cluster 1 & 0.6766 & 0.6634 & 7.1659 & 2.4167 & 9.1031 & 3.0133 \\
\hline Cluster 2 & -0.0500 & 0.0035 & 2.6492 & 3.7953 & 35.2965 & 53.7835 & Cluster 2 & 0.1584 & 0.1542 & 0.1725 & 0.1306 & 1.5800 & 2.6557 \\
\hline Cluster 3 & -2.2598 & -2.2513 & 3.9292 & 4.9389 & 102.5378 & 119.4613 & Cluster 3 & 0.1901 & 0.2174 & 0.2859 & 0.2473 & 1.6885 & 1.9154 \\
\hline Cluster 4 & -12.4734 & -12.6754 & -4.8020 & -4.3008 & 139.0158 & 148.3612 & Cluster 4 & 0.1601 & 0.2154 & 0.2733 & 0.2348 & 1.3228 & 0.9242 \\
\hline \multicolumn{7}{|c|}{ Coordinate 2 (spin plane) } & \multicolumn{7}{|c|}{ Coordinate 2 (spin plane) } \\
\hline & Range 2 & Range 3 & Range 4 & Range 5 & Range 6 & Range 7 & & Range 2 & Range 3 & Range 4 & Range 5 & Range 6 & Range 7 \\
\hline Cluster 1 & 6.3769 & 6.5161 & 23.5516 & 27.1099 & 290.7744 & 306.5910 & Cluster 1 & 0.8277 & 0.8612 & 2.9593 & 1.1104 & 4.6518 & 0.7906 \\
\hline Cluster 2 & -2.4250 & -2.4272 & -2.3178 & -1.9328 & -9.1467 & -1.4474 & Cluster 2 & 0.1352 & 0.1352 & 0.1721 & 0.1973 & 1.8764 & 0.4969 \\
\hline Cluster 3 & -5.0619 & -5.0892 & 1.4972 & 2.5119 & 107.5918 & 123.1910 & Cluster 3 & 0.1608 & 0.1805 & 0.2780 & 0.1713 & 1.4813 & 1.1673 \\
\hline Cluster 4 & -3.0896 & -3.0821 & 3.4234 & 4.4197 & 118.6630 & 136.1260 & Cluster 4 & 0.1226 & 0.1301 & 0.1916 & 0.2587 & 1.8519 & 1.5174 \\
\hline \multicolumn{7}{|c|}{ Coordinate 3 (spin plane) } & \multicolumn{7}{|c|}{ Coordinate 3 (spin plane) } \\
\hline & Range 2 & Range 3 & Range 4 & Range 5 & Range 6 & Range 7 & & Range 2 & Range 3 & Range 4 & Range 5 & Range 6 & Range 7 \\
\hline Cluster 1 & 0.3404 & 0.3902 & -3.7819 & -4.2530 & -45.3345 & -30.1651 & Cluster 1 & 0.4079 & 0.4161 & 1.4741 & 0.7083 & 2.2685 & 0.4807 \\
\hline Cluster 2 & -1.3266 & -1.2950 & -0.7289 & -0.2210 & -0.3983 & 9.7329 & Cluster 2 & 0.1898 & 0.1944 & 0.2567 & 0.2281 & 1.5767 & 0.8230 \\
\hline Cluster 3 & -2.5431 & -2.5589 & 2.4849 & 3.0053 & 90.8152 & 101.5091 & Cluster 3 & 0.1376 & 0.1408 & 0.2026 & 0.1633 & 2.4599 & 1.8600 \\
\hline Cluster 4 & 4.4047 & 4.5053 & 12.7467 & 13.3045 & 156.8103 & 166.8933 & Cluster 4 & 0.1780 & 0.1866 & 0.1862 & 0.1681 & 1.8072 & 1.2829 \\
\hline
\end{tabular}

Table 3. Mean and standard deviation of gains $\left(G_{1}\right.$ and $\left.\Delta G_{32}\right)$ for the mission segment from February 2001 to February 2012 for each coordinate in every range on all spacecraft. Standard deviations for ranges that are at least twice as large as those for all the other spacecraft in that range are highlighted in italic.

\begin{tabular}{|c|c|c|c|c|c|c|c|c|c|c|c|c|c|}
\hline \multicolumn{14}{|c|}{ Gain } \\
\hline \multicolumn{7}{|c|}{ Mean } & \multicolumn{7}{|c|}{ Standard deviation } \\
\hline \multicolumn{7}{|c|}{ Coordinate 1 (spin axis) } & \multicolumn{7}{|c|}{ Coordinate 1 (spin axis) } \\
\hline & Range 2 & Range 3 & Range 4 & Range 5 & Range 6 & Range 7 & & Range 2 & Range 3 & Range 4 & Range 5 & Range 6 & Range 7 \\
\hline Cluster 1 & 0.9501 & 0.9684 & 0.9795 & 0.9962 & 0.9768 & 0.9978 & Cluster 1 & 0.0037 & 0.0024 & 0.0027 & 0.0001 & 0.0046 & 0.0030 \\
\hline Cluster 2 & 0.9589 & 0.9759 & 0.9866 & 1.0034 & 0.9853 & 1.0012 & Cluster 2 & 0.0017 & 0.0007 & 0.0010 & 0.0001 & 0.0027 & 0.0007 \\
\hline Cluster 3 & 0.9601 & 0.9756 & 0.9954 & 1.0110 & 0.9954 & 1.0106 & Cluster 3 & 0.0030 & 0.0010 & 0.0010 & 0.0001 & 0.0068 & 0.0008 \\
\hline Cluster 4 & 0.9595 & 0.9783 & 0.9954 & 1.0130 & 0.9925 & 1.0108 & Cluster 4 & 0.0032 & 0.0008 & 0.0002 & 0.0001 & 0.0010 & 0.0019 \\
\hline \multicolumn{7}{|c|}{ Coordinate 3 - Coordinate 2 (spin plane) } & \multicolumn{7}{|c|}{ Coordinate 3 - Coordinate 2 (spin plane) } \\
\hline & Range 2 & Range 3 & Range 4 & Range 5 & Range 6 & Range 7 & & Range 2 & Range 3 & Range 4 & Range 5 & Range 6 & Range 7 \\
\hline Cluster 1 & 0.0157 & 0.0151 & 0.0108 & 0.0101 & 0.0084 & 0.0071 & Cluster 1 & 0.0008 & 0.0003 & 0.0001 & 0.0000 & 0.0000 & 0.0014 \\
\hline Cluster 2 & -0.0057 & -0.0066 & 0.0041 & 0.0031 & 0.0049 & 0.0040 & Cluster 2 & 0.0007 & 0.0003 & 0.0002 & 0.0000 & 0.0000 & 0.0000 \\
\hline Cluster 3 & -0.0180 & -0.0163 & -0.0130 & -0.0112 & -0.0104 & -0.0085 & Cluster 3 & 0.0004 & 0.0003 & 0.0001 & 0.0001 & 0.0001 & 0.0001 \\
\hline Cluster 4 & 0.0246 & 0.0240 & 0.0266 & 0.0259 & 0.0237 & 0.0230 & Cluster 4 & 0.0004 & 0.0003 & 0.0001 & 0.0001 & 0.0002 & 0.0002 \\
\hline
\end{tabular}

Additionally, a preliminary attempt has been made to correlate variation in the calibration parameters with instrument housekeeping telemetry. The instrument housekeeping consists of the following quantities: the FGM electronics box temperature located within the body of the spacecraft, the FGM outboard and inboard sensor temperatures on the boom, and the currents and the voltages of the electronics inside the electronics box. Only the temperatures are discussed here.

\subsection{Cross-spacecraft comparisons of instrument housekeeping telemetry values}

The data displayed in Figs. 2 and 3 are for the outboard sensors on all of the spacecraft. They cover the period from February 2001 (orbit 93) to August 2012 (orbit 1889).

\subsubsection{FGM outboard sensor temperature}

The FGM sensors each contain a thermistor, which is independent of the FGM electronics and which is monitored by the spacecraft. Each sensor also contains a heater which can be operated independently from the remainder of the 

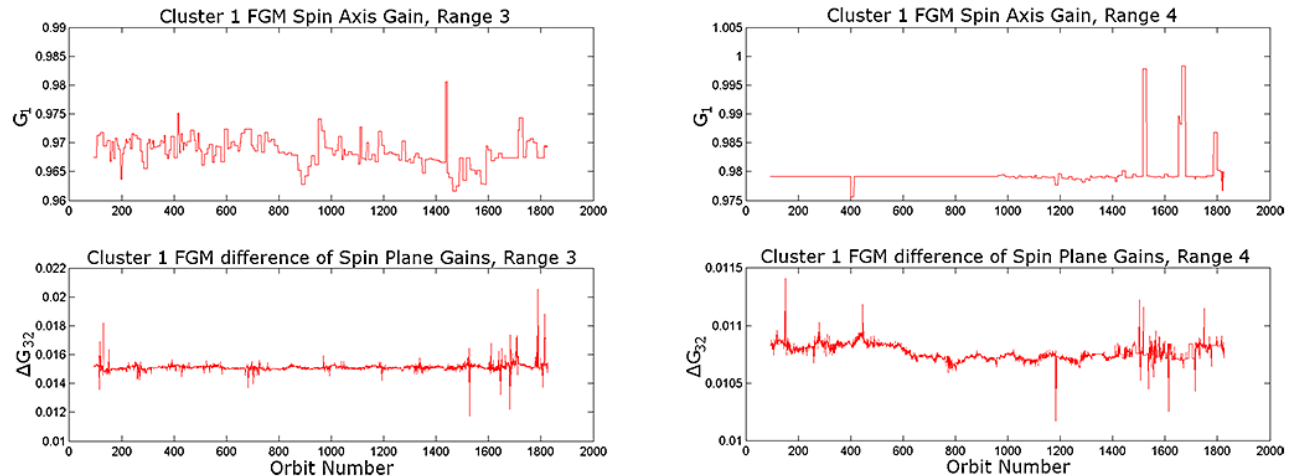

Figure 10. Gain plots $\left(G_{1}\right.$ and $\left.\Delta G_{32}\right)$ from February 2001 to February 2012 for exceptional cases in $\mathrm{C} 1$.
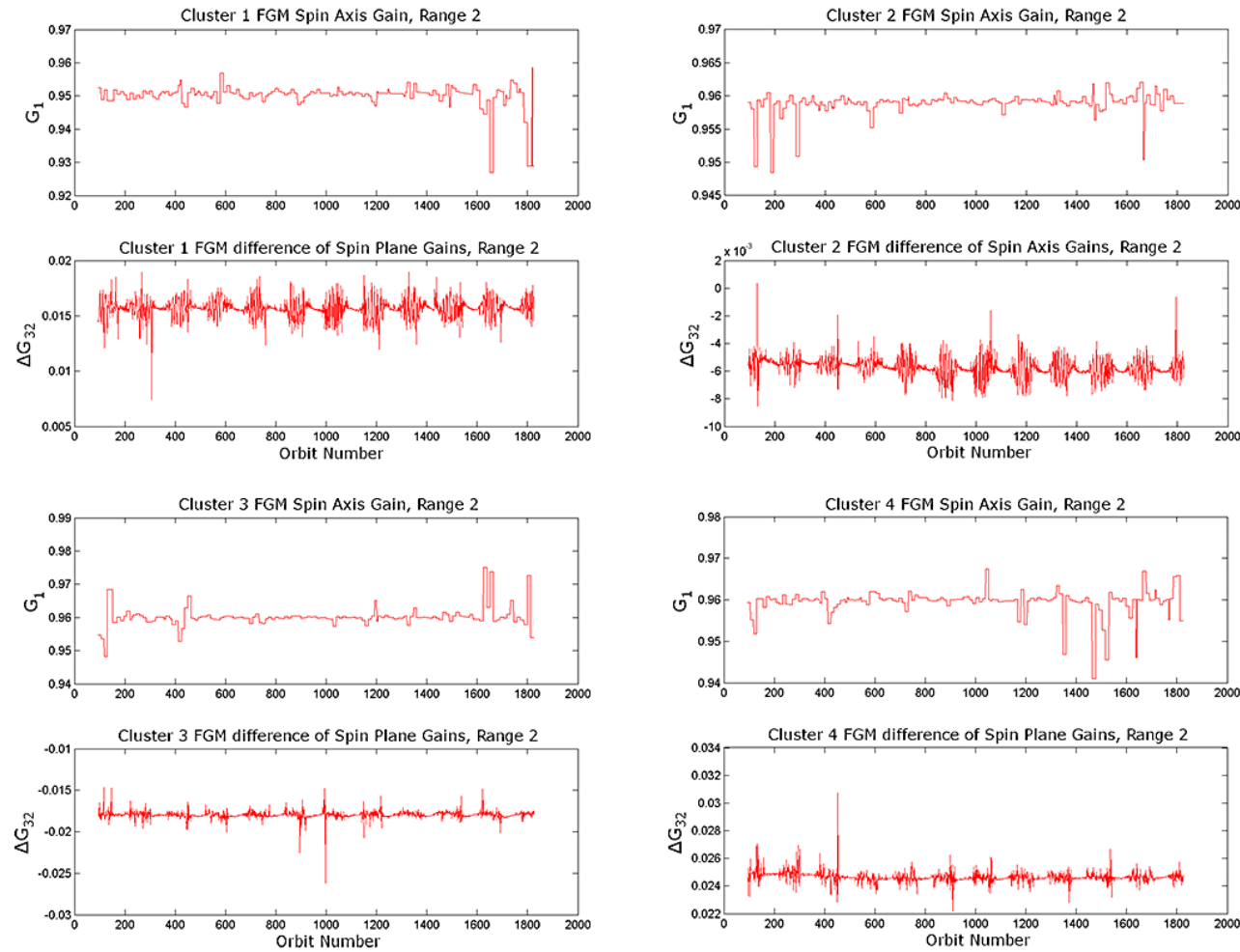

Figure 11. Gain plots $\left(G_{1}\right.$ and $\left.\Delta G_{32}\right)$ from February 2001 to February 2012 showing periodic behaviour of spin-plane gain difference for range 2 in all spacecraft.

spacecraft electronics. Both heaters are powered through a single switch which is controlled by the spacecraft. The sensors have thermal insulation and their temperature can be expected to change at a maximum of $20^{\circ} \mathrm{Ch}^{-1}$. The sensors are monitored at intervals of the order of $30 \mathrm{~min}$ (FGM instrument users manual). Although each of these values is monitored more frequently, a single averaged value has been shown for each orbit (51-57 h) in Fig. 2.

The outboard sensor temperatures for all four spacecraft show a cyclical fluctuation over the course of nightside-todayside transitions, becoming around $5^{\circ} \mathrm{C}$ warmer during the peak of the dayside season. The spikes are due to long eclipse periods, during which the FGM is off. All sensors have undergone a warming trend over the course of the mission as shown in Fig. 2. Since the outboard FGM sensors are located on the ends of $5 \mathrm{~m}$ booms, the warming and cooling cycle is most likely related to the spacecrafts' positions relative to the Sun during dayside and nightside seasons. The overall warming trend is likely related to the spacecrafts' positions relative to the Earth, as both periapsis and apoapsis have become lower over the course of the mission. 

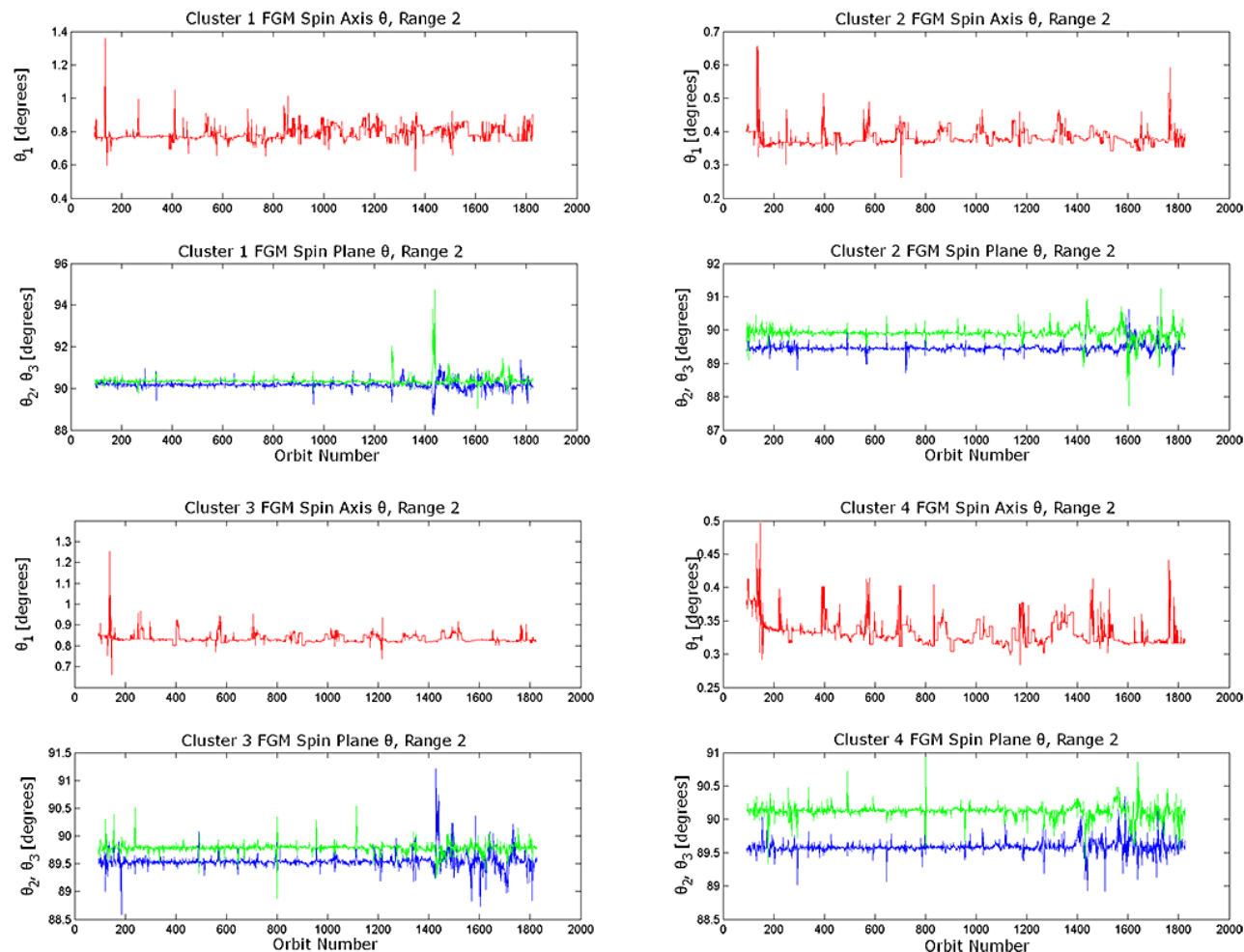

Figure 12. Elevation angle, $\theta_{i}$, plots from February 2001 to February 2012 for range 2 for all spacecraft.

\subsubsection{FGM electronics box temperature}

The electronics box temperature is monitored from a thermistor located on the DC-DC converter card. This temperature can be expected to follow the temperature of the main equipment platform. The upper and lower operational limits for the box temperature are $+60^{\circ} \mathrm{C}$ and $-25^{\circ} \mathrm{C}$. If these limits are exceeded, the FGM instrument is powered off (Brown et al., 2000). Although each of these values is monitored more frequently, a single averaged value has been shown for each orbit (51-57 h) in Fig. 3.

On all four spacecraft, the box temperature is about $21^{\circ} \mathrm{C}$, with a declining trend until around orbit 600 (late May 2004), when it becomes cyclical as seen in the outboard sensor temperature. The boxes undergo less dramatic warming than the outboard sensors of around $3{ }^{\circ} \mathrm{C}$ during the peak of the dayside season. This is likely due to their less exposed positions on the spacecraft body. The electronics boxes appear to be cooling over the course of the mission, with $\mathrm{C} 1$ and $\mathrm{C} 3$ cooling less dramatically than $\mathrm{C} 2$ and $\mathrm{C} 4$. The electronics boxes, due to their position on the spacecraft platform, are coupled to the spacecraft temperature much more strongly than the boom-mounted units. Changes in spacecraft heating strategy over the course of the mission, with more portions of the spacecraft being turned off during eclipses, are reflected in the electronics box temperature behaviour.

\subsection{Inter-spacecraft calibration parameter comparisons by range}

The data displayed are for the outboard sensors on all of the spacecraft and run from February 2001 (orbit 93) to February 2012 (orbit 1825).

\subsubsection{Offsets}

In Figs. 4 through 9, the individual offsets have been plotted for each range and spacecraft. The offsets in red have been applied to the spin-axis component of the magnetic field vector, while those in green and blue have been applied to the spin-plane components of the magnetic field vector. One offset value is applied across all data for a given range in an orbit. The spin-plane offsets are adjusted on a per-orbit basis no matter the phase of the mission. The spin-axis offsets, as mentioned in the Introduction, are adjusted on a biweekly or monthly basis during the dayside season when the spacecraft are in the solar wind and then interpolated between the end of one dayside season and the start of the next. The biweekly/monthly adjustment of the spin-axis offset gives a short, step-like appearance to the offset lines, while the interpolation method gives longer sloping steps for the 7 or so months (around 100 orbits) that the spacecraft spend on the nightside portion of their tours.

Comparison of the smooth slopes of the interpolated offsets with the variability of the solar wind-adjusted offsets 
Table 4. Mean and standard deviation of elevation angles $\left(\theta_{i}\right)$ for the mission segment from February 2001 to February 2012 in every range on all spacecraft. Standard deviations for ranges that are at least twice as large as those for all the other spacecraft in that range are highlighted.

\begin{tabular}{|c|c|c|c|c|c|c|c|c|c|c|c|c|c|}
\hline \multicolumn{14}{|c|}{ Theta $\left({ }^{\circ}\right)$} \\
\hline \multicolumn{7}{|c|}{ Mean } & \multicolumn{7}{|c|}{ Standard deviation } \\
\hline \multicolumn{7}{|c|}{ Coordinate 1 (spin axis) } & \multicolumn{7}{|c|}{ Coordinate 1 (spin axis) } \\
\hline & Range 2 & Range 3 & Range 4 & Range 5 & Range 6 & Range 7 & & Range 2 & Range 3 & Range 4 & Range 5 & Range 6 & Range 7 \\
\hline Cluster 1 & 0.7878 & 0.7711 & 0.7696 & 0.7736 & 0.7585 & 0.7527 & Cluster 1 & 0.0436 & 0.0123 & 0.0075 & 0.0064 & 0.0053 & 0.0142 \\
\hline Cluster 2 & 0.3806 & 0.3669 & 0.3674 & 0.3709 & 0.3645 & 0.3582 & Cluster 2 & 0.0238 & 0.0059 & 0.0049 & 0.0029 & 0.0038 & 0.0035 \\
\hline Cluster 3 & 0.8343 & 0.8232 & 0.8242 & 0.8223 & 0.8270 & 0.8268 & Cluster 3 & 0.0226 & 0.0073 & 0.0036 & 0.0026 & 0.0019 & 0.0017 \\
\hline Cluster 4 & 0.3319 & 0.3216 & 0.3352 & 0.3301 & 0.3564 & 0.3445 & Cluster 4 & 0.0195 & 0.0102 & 0.0083 & 0.0048 & 0.0084 & 0.0029 \\
\hline \multicolumn{7}{|c|}{ Coordinate 2 (spin plane) } & \multicolumn{7}{|c|}{ Coordinate 2 (spin plane) } \\
\hline & Range 2 & Range 3 & Range 4 & Range 5 & Range 6 & Range 7 & & Range 2 & Range 3 & Range 4 & Range 5 & Range 6 & Range 7 \\
\hline Cluster 1 & 90.1757 & 90.1731 & 90.1919 & 90.1666 & 90.1940 & 90.2105 & Cluster 1 & 0.1831 & 0.2995 & 0.0522 & 0.0160 & 0.0144 & 0.0044 \\
\hline Cluster 2 & 89.4633 & 89.4650 & 89.4575 & 89.4573 & 89.4710 & 89.4661 & Cluster 2 & 0.1162 & 0.1332 & 0.0180 & 0.0080 & 0.0232 & 0.0059 \\
\hline Cluster 3 & 89.5477 & 89.5165 & 89.5268 & 89.5276 & 89.5205 & 89.5174 & Cluster 3 & 0.1426 & 0.1646 & 0.0147 & 0.0060 & 0.0178 & 0.0122 \\
\hline Cluster 4 & 89.5849 & 89.5746 & 89.5695 & 89.5703 & 89.5654 & 89.5660 & Cluster 4 & 0.0920 & 0.1468 & 0.0139 & 0.0088 & 0.0173 & 0.0056 \\
\hline \multicolumn{7}{|c|}{ Coordinate 3 (spin plane) } & \multicolumn{7}{|c|}{ Coordinate 3 (spin plane) } \\
\hline & Range 2 & Range 3 & Range 4 & Range 5 & Range 6 & Range 7 & & Range 2 & Range 3 & Range 4 & Range 5 & Range 6 & Range 7 \\
\hline Cluster 1 & 90.3697 & 90.3718 & 90.3484 & 90.3567 & 90.3604 & 90.3438 & Cluster 1 & 0.2282 & 0.3353 & 0.0355 & 0.0096 & 0.0087 & 0.0103 \\
\hline Cluster 2 & 89.9023 & 89.8828 & 89.9163 & 89.9048 & 89.9416 & 89.9405 & Cluster 2 & 0.1784 & 0.2616 & 0.0290 & 0.0122 & 0.0226 & 0.0071 \\
\hline Cluster 3 & 89.7856 & 89.7890 & 89.7895 & 89.7908 & 89.7961 & 89.7948 & Cluster 3 & 0.0852 & 0.0936 & 0.0147 & 0.0046 & 0.0204 & 0.0143 \\
\hline Cluster 4 & 90.1174 & 90.1101 & 90.1471 & 90.1448 & 90.1573 & 90.1641 & Cluster 4 & 0.1012 & 0.1981 & 0.0190 & 0.0093 & 0.0157 & 0.0032 \\
\hline
\end{tabular}

Table 5. Mean and standard deviation of azimuthal angles $\left(\varphi_{1}\right.$ and $\left.\Delta \varphi_{32}\right)$ for the segment from February 2001 to February 2012 in every range on all spacecraft. Standard deviations for ranges that are at least twice as large as those for all the other spacecraft in that range are highlighted in italic.

\begin{tabular}{|c|c|c|c|c|c|c|c|c|c|c|c|c|c|}
\hline \multicolumn{14}{|c|}{$\operatorname{Phi}\left({ }^{\circ}\right)$} \\
\hline \multicolumn{7}{|c|}{ Mean } & \multicolumn{7}{|c|}{ Standard deviation } \\
\hline \multicolumn{7}{|c|}{ Coordinate 1 (spin axis) } & \multicolumn{7}{|c|}{ Coordinate 1 (spin axis) } \\
\hline & Range 2 & Range 3 & Range 4 & Range 5 & Range 6 & Range 7 & & Range 2 & Range 3 & Range 4 & Range 5 & Range 6 & Range 7 \\
\hline Cluster 1 & -119.1486 & -119.7610 & -119.9031 & -119.3920 & -120.2991 & -120.1796 & Cluster 1 & 3.0070 & 1.1482 & 0.7069 & 0.3514 & 0.4094 & 0.8647 \\
\hline Cluster 2 & -64.0441 & -64.6107 & -64.7980 & -64.0815 & -63.5844 & -62.9304 & Cluster 2 & 3.1663 & 1.5004 & 1.0614 & 0.5710 & 0.6295 & 0.5848 \\
\hline Cluster 3 & 167.4990 & 167.3238 & 167.4863 & 167.3277 & 167.6714 & 167.6328 & Cluster 3 & 1.0820 & 0.4848 & 0.4424 & 0.2880 & 0.1726 & 0.0572 \\
\hline Cluster 4 & -89.3487 & -89.6470 & -89.6600 & -88.7984 & -88.5335 & -88.1003 & Cluster 4 & 3.0770 & 2.2709 & 1.3110 & 1.0266 & 0.5088 & 0.3284 \\
\hline \multicolumn{7}{|c|}{ Coordinate 3 - Coordinate 2 (spin plane) } & \multicolumn{7}{|c|}{ Coordinate 3 - Coordinate 2 (spin plane) } \\
\hline & Range 2 & Range 3 & Range 4 & Range 5 & Range 6 & Range 7 & & Range 2 & Range 3 & Range 4 & Range 5 & Range 6 & Range 7 \\
\hline Cluster 1 & 89.7586 & 89.7502 & 89.5435 & 89.5716 & 89.5199 & 89.5177 & Cluster 1 & 0.0953 & 0.0869 & 0.0312 & 0.0083 & 0.0027 & 0.0009 \\
\hline Cluster 2 & 89.3710 & 89.3698 & 89.3617 & 89.3659 & 89.3692 & 89.3635 & Cluster 2 & 0.0301 & 0.0208 & 0.0075 & 0.0059 & 0.0072 & 0.0039 \\
\hline Cluster 3 & 89.2561 & 89.2549 & 89.3181 & 89.3183 & 89.3300 & 89.3261 & Cluster 3 & 0.0287 & 0.0101 & 0.0033 & 0.0032 & 0.0031 & 0.0018 \\
\hline Cluster 4 & 88.8318 & 88.8325 & 88.8201 & 88.8252 & 88.8267 & 88.8236 & Cluster 4 & 0.0232 & 0.0157 & 0.0086 & 0.0054 & 0.0052 & 0.0045 \\
\hline
\end{tabular}

shows that the interpolation is probably masking the natural variability during the tail season. Orbits for which no FGM data were taken (and hence no calibration) have been omitted.

Over the course of the mission, offset drift is negligible in all components. The largest drifts in offsets take place on $\mathrm{C} 1$. $\mathrm{C} 4$ is the only other spacecraft with offsets that are comparable in magnitude to $\mathrm{C} 1$ in ranges 2 through 5, but offset drift is still insignificant. Offset variation with temperature is about $0.2 \mathrm{nT}^{\circ} \mathrm{C}^{-1}$ on $\mathrm{C} 1$ and $0.1 \mathrm{nT} \mathrm{C}^{-1}$ on $\mathrm{C} 2, \mathrm{C} 3$ and $\mathrm{C} 4$.

For $\mathrm{C} 1$ in range 2 (Fig. 4) there is a clear decreasing trend in the spin-axis offset $O_{1}$, which is visible even with the steps introduced by the interpolated values over the course of the mission. A decreasing trend is also seen in $\mathrm{O}_{3}$. The total change is about $2 \mathrm{nT}$ in $\mathrm{O}_{1}$ and $1 \mathrm{nT}$ in $\mathrm{O}_{3}$. In $\mathrm{O}_{2}$, the offset appears to increase by about $2 \mathrm{nT}$. On the other spacecraft, $\mathrm{C} 2-\mathrm{C} 4$, there is no overall drift, although some cyclical behaviour that may be related to instrument parameter cycles, particularly in the outboard sensor temperature, can be seen in $\mathrm{C} 2$ and $\mathrm{C} 3$.

Range 3 offset trends are very similar in magnitude and type to those observed in range 2 (Fig. 5). This is not too surprising since the range change is achieved by switching a single feedback resistor. On $\mathrm{C} 1$, the decreases of $2 \mathrm{nT}$ in the spin-axis offset $O_{1}$ and $1 \mathrm{nT}$ in $O_{3}$ are observed, as is the 2 nT increase in $\mathrm{O}_{2}$. On the other spacecraft, $\mathrm{C} 2-\mathrm{C} 4$, there is 

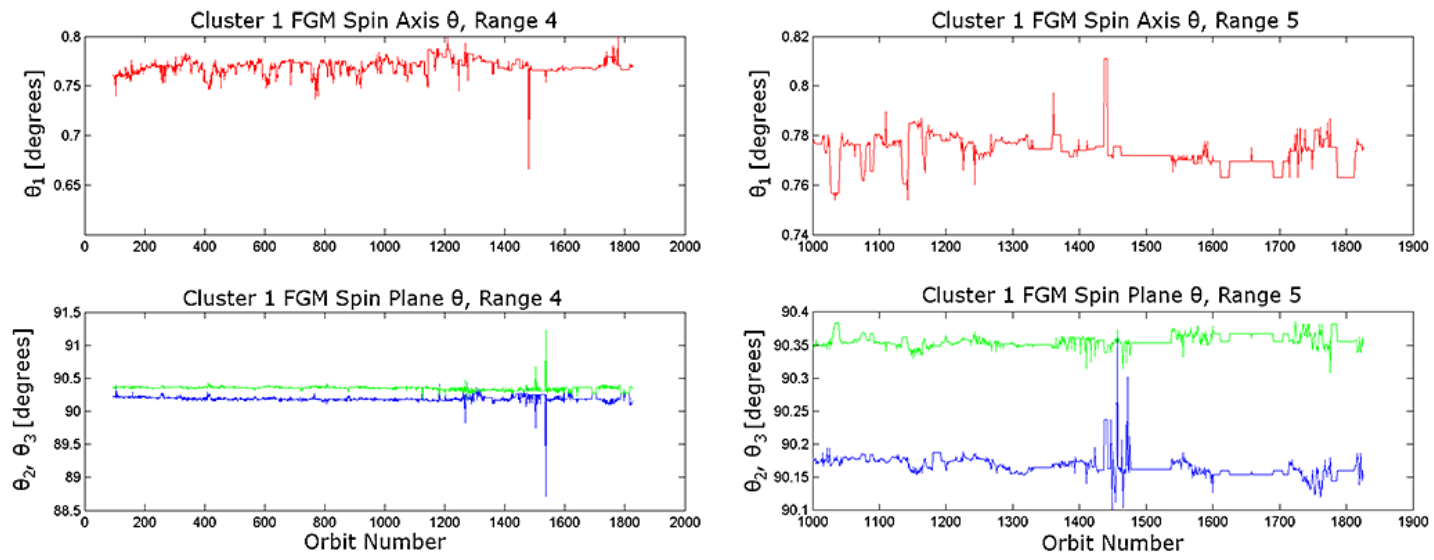

Figure 13. Elevation angle, $\theta_{i}$, plots for C1, ranges 4 and 5, from February 2001 to February 2012.
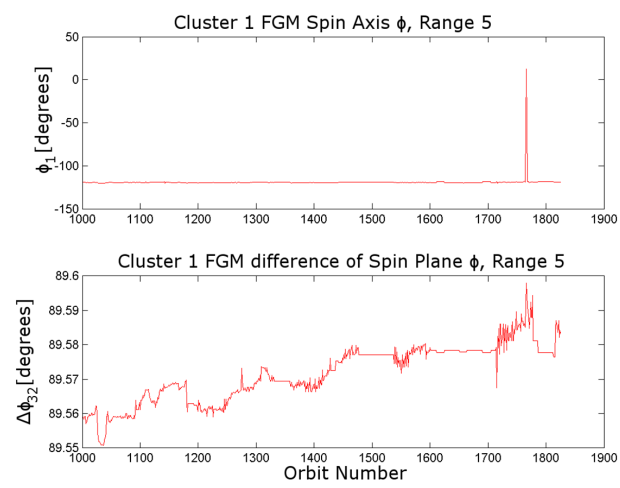

(a)
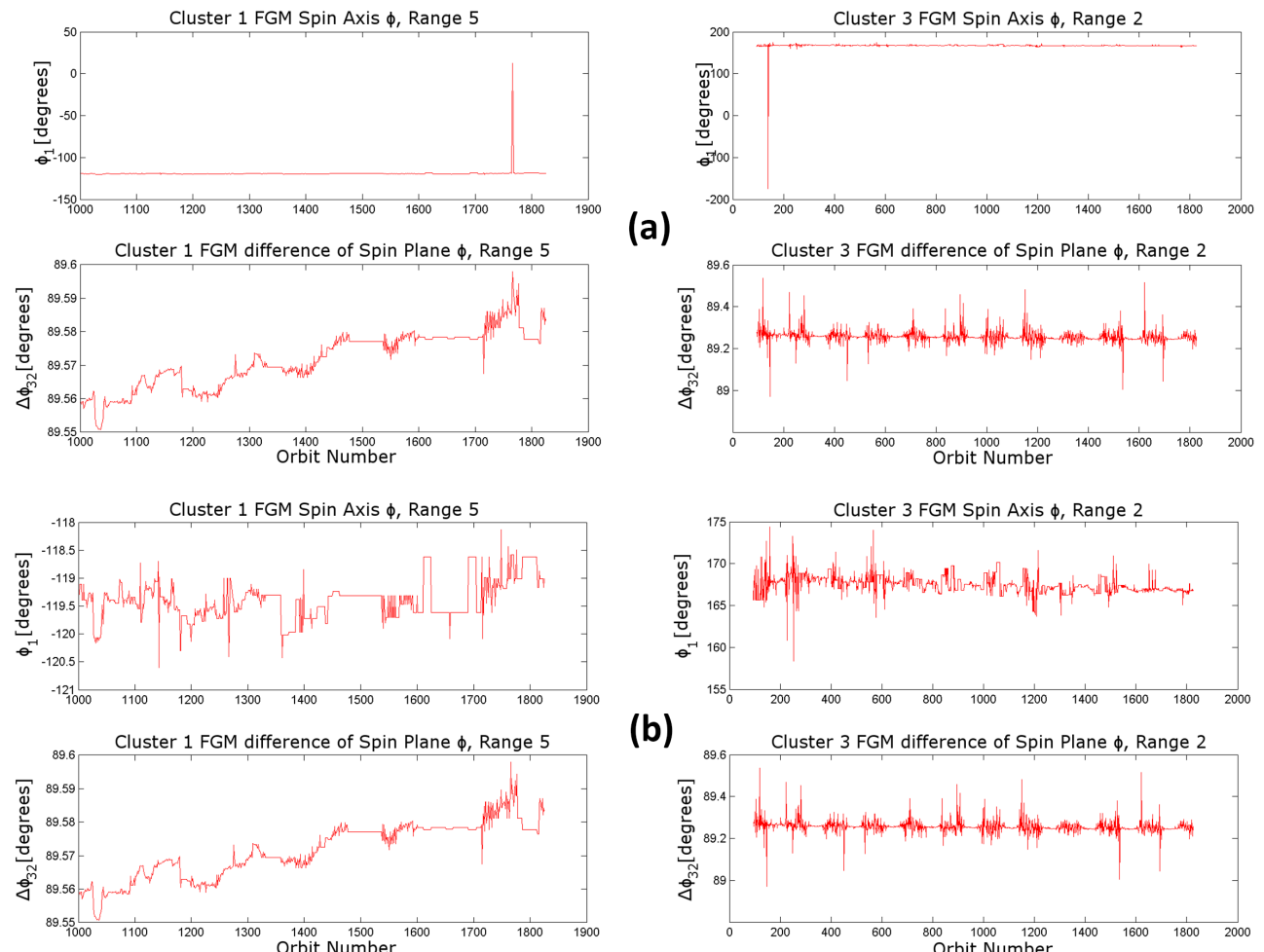

(b)

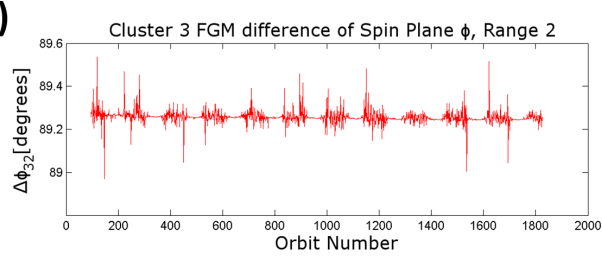

Figure 14. Azimuthal angles, $\varphi_{1}$ and $\Delta \varphi_{32}$, for $\mathrm{C} 1$, range 5 and $\mathrm{C} 3$, range 2 . The top four plots show the angles before the unphysical calibration parameters were corrected and the lower four plots show them after correction.

no overall drift, although some cyclical behaviour that may be related to instrument parameter cycles, particularly in the outboard sensor temperature, can be seen in $\mathrm{C} 2$ and $\mathrm{C} 3$. The offsets also appear to fluctuate more, particularly the spinaxis offset, early in the mission compared to range 2 . Some outlying values in the spin-axis offset for $\mathrm{C} 2$ will require further investigation.

Range 4 offset trends are similar in type to those observed in range 2 (Fig. 6). The $\mathrm{C} 1$ drifts have increased by approximately an order of magnitude. The decrease of $2 \mathrm{nT}$ has be- come $20 \mathrm{nT}$ in the spin-axis offset $O_{1}$ and $1 \mathrm{nT}$ in $O_{3}$ has become $8 \mathrm{nT}$. The $2 \mathrm{nT}$ increase in $O_{2}$ has become $15 \mathrm{nT}$. On the other spacecraft, $\mathrm{C} 2-\mathrm{C} 4$, there is no overall drift, although some cyclical behaviour that may be related to instrument parameter cycles, particularly in the outboard sensor temperature, can be seen on $\mathrm{C} 2$ and $\mathrm{C} 3$. The offsets fluctuate less, particularly the spin-axis offset, early in the mission compared to range 3 .

Since range 5 did not enter routine use until late November 2006, Fig. 7 covers 800 orbits, or just over 5 years. C1 

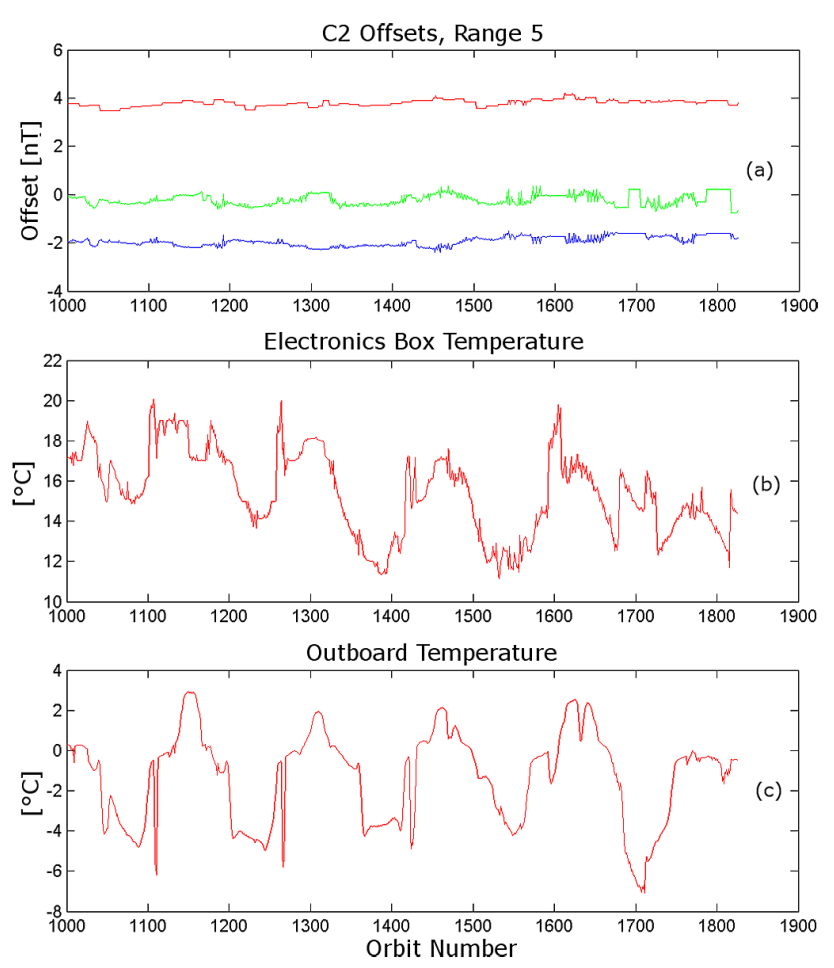

Figure 15. (a) $\mathrm{C} 2$ range 5 offsets $\left(O_{1}\right.$ in red, $O_{2}$ in blue and $\mathrm{O}_{3}$ in green), (b) $\mathrm{C} 2$ electronics box temperature and (c) $\mathrm{C} 2$ outboard sensor temperature. Offsets shown in nanoteslas and temperatures in degrees Celsius for orbits 1000-1812 (December 2006February 2012).

follows the trend seen in the lower ranges, where $O_{1}$ and $O_{3}$ are slowly decreasing and $\mathrm{O}_{2}$ is slowly increasing. $\mathrm{C} 2$ shows slight signs of a cyclical trend like that observed in the outboard sensor and box temperatures. C3 shows strong signs of such a cyclical trend, while $\mathrm{C} 4$ shows the same stability and independence of instrument parameter trends exhibited previously. The potential correlation with instrument parameters in $\mathrm{C} 2$ and $\mathrm{C} 3$ merits further investigation in another section of the Analysis.

Despite the limited data available in range 6, the offset trends mirror those seen in the range 5 data (Fig. 8). At present there are 500 orbits' worth, or just over 3 years, of data. As discussed in the Introduction, limited ground calibration information was available for this range since it was not originally intended for scientific investigation. Changes in the range 6 parameters, including the offsets, are tied to changes in the range 5 parameters, which were used during the initial calibration of range 6 to help discover consistent values. It is therefore sensible that any potential correlation with other instrument parameters that were seen in range 5 should also be observed in range 6 . As the mission continues and range 6 is employed more regularly for lower periapsis passes, the consistency between range 5 and range 6 offsets should become clearer.
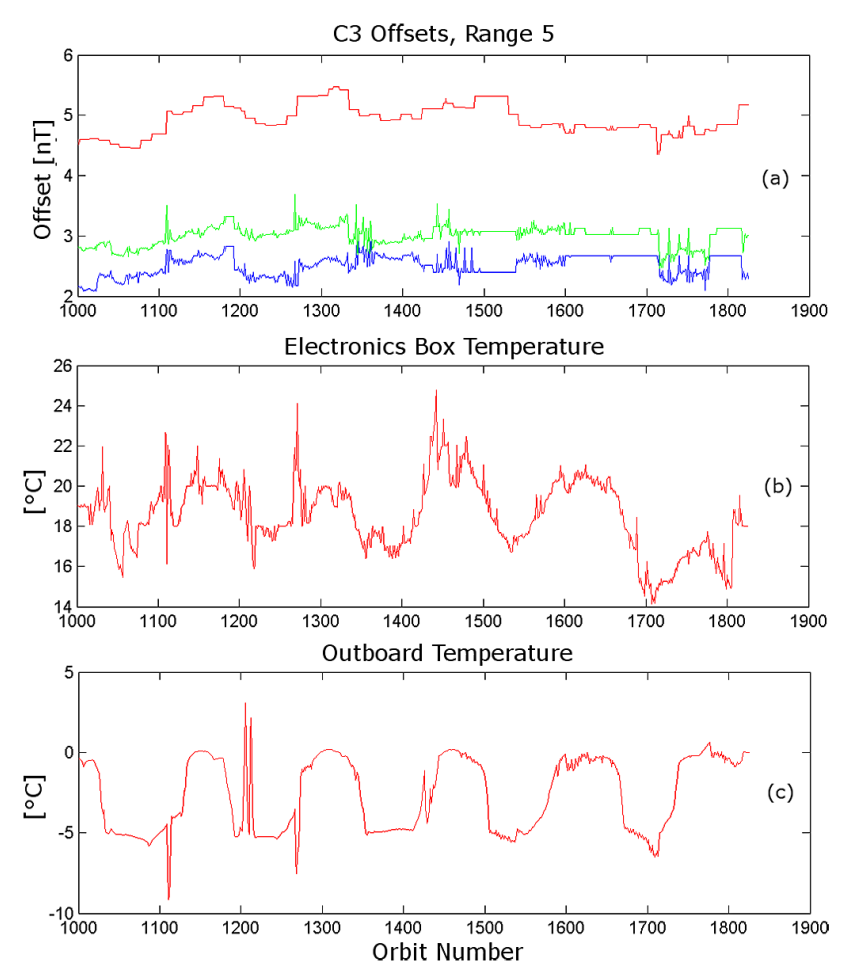

Figure 16. (a) $\mathrm{C} 3$ range 5 offsets $\left(O_{1}\right.$ in red, $O_{2}$ in blue and $\mathrm{O}_{3}$ in green), (b) $\mathrm{C} 3$ electronics box temperature and (c) $\mathrm{C} 3$ outboard sensor temperature. Offsets shown in nanoteslas and temperatures in degrees Celsius for orbits 1000-1825 (December 2006February 2012).

Insufficient data exists in range 7 to distinguish many trends (Fig. 9). One exception is that large month-long deviations in a parameter, such as the one seen in the spin-axis offset around orbit 1600 in C3, are paralleled by similar deviations in range 6 . Adjustments to the spin-axis offsets during range jump corrections are primarily responsible for such shifts, since a large change in the range 6 spin-axis offset to eliminate R56 jumps is likely to result in the need for a large change in the range 7 spin-axis offset to eliminate the R67 jumps.

At most (on $\mathrm{C} 2$ and $\mathrm{C} 4$ ) there are 60 orbits' worth, or 5 months, of data and at least (on C1) there are 36 orbits' worth, or 3 months, of data. Since the spacecraft are now off due to power-sharing issues during the lower periapsis passes that necessitated the use of range 7, it is unlikely that this limited set will be expanded much. It will therefore not be possible to determine whether range 7 follows the same trends as observed in the lower ranges for each spacecraft. Further discussion of this range has been omitted from the remainder of this article.

In Table 2, the mean value and standard deviations for the spin-axis and spin-plane offsets on each spacecraft over orbits 93-1825 (February 2001-February 2012) have been calculated. The standard deviations in this, and in Tables 3- 

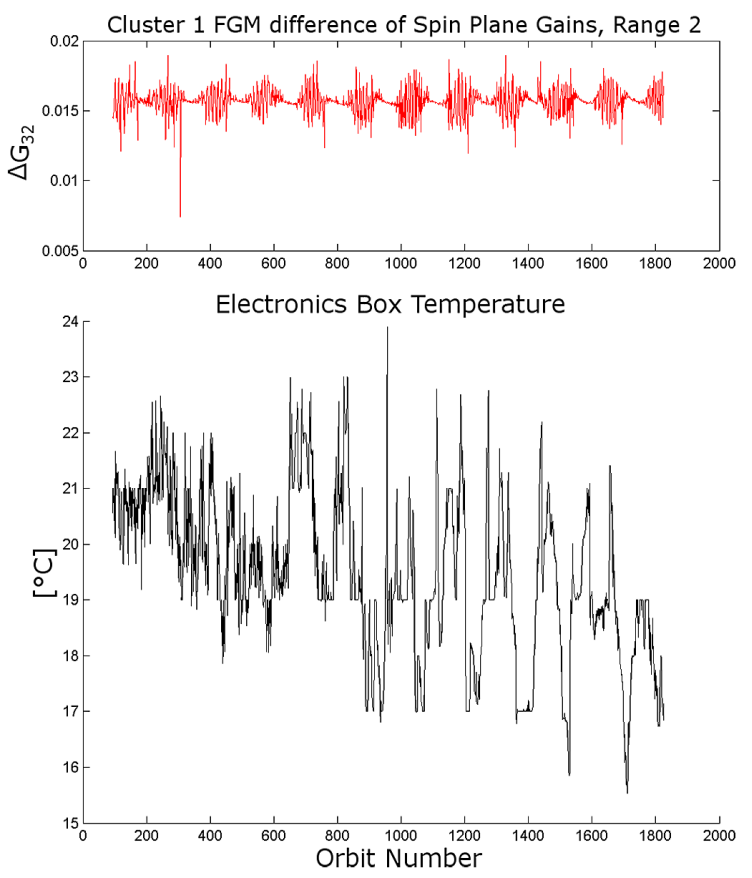

Figure 17. Spin-plane gain difference $\left(\Delta G_{32}\right)$ for $\mathrm{C} 1$, range 2 and electronics box temperatures in degrees Celsius for orbits 93-1825 (February 2001-February 2012).

5 , should be viewed as a measure of the variability in the output of the calibration procedures and not necessarily as a measure of physical variability in the sensor itself. The standard deviations are fairly consistent between coordinates and across all ranges for $\mathrm{C} 2, \mathrm{C} 3$ and $\mathrm{C} 4$. With the exception of range 7 , the standard deviation for $\mathrm{C} 1$ is significantly larger, from two up to thirty times greater than the other spacecraft. This tallies with the observation of greater long-term drift in the offset parameters for $\mathrm{C} 1$ than in the other spacecraft.

\subsubsection{Gains and angles}

For most of the remaining calibration parameters, the fluctuations across the mission show no visible correlation with instrument parameters and no long-term trends. Mission averages for the parameters, which are the gains $\left(G_{i}\right)$ and angles $\left(\theta_{i}\right.$ and $\left.\varphi_{i}\right)$ will therefore be discussed in tabular form, with plots shown for exceptional cases.

In Table 3, the mean value and standard deviations for the spin-axis gain $\left(G_{1}\right)$ and the difference of the spin-plane gains $\left(\Delta G_{32}\right)$ have been calculated. The change in the difference between the spin-plane gains is used as a calibration parameter. The final spin-plane gain values are therefore interdependent, which is why the difference is evaluated here. With two exceptions, notably the spin-axis gains for ranges 3 and 4 on $\mathrm{C} 1$, there is little fluctuation in these parameters. The noted gains are shown in Fig. 10.

The two exceptional cases show similar behaviour. In ranges 3 and 4 of $\mathrm{C} 1$, the fluctuations begin in orbits cor-
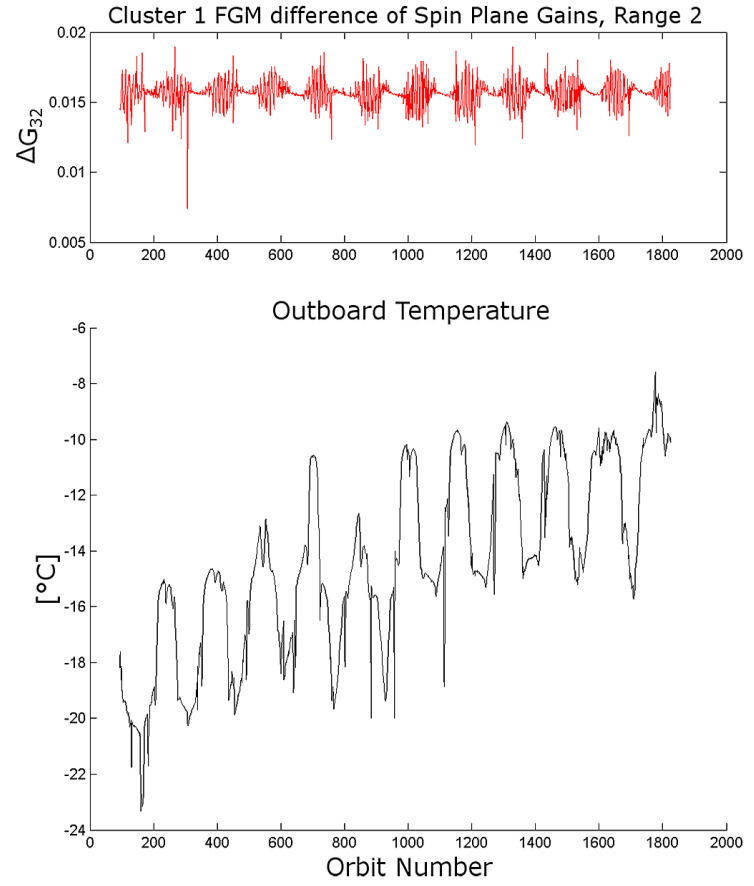

Figure 18. Spin-plane gain difference $\left(\Delta G_{32}\right)$ for $\mathrm{C} 1$, range 2 and sensor temperatures in degrees Celsius for orbits 93-1825 (February 2001-February 2012).

responding to late 2010 , with no obvious correlation to other behaviour, through to February 2012. The calibration for the orbits where large, probably non-physical fluctuation in the gain is seen will need to be revised.

One interesting behaviour not reflected in the gains table of averages and standard deviations occurs in range 2 for all spacecraft. The spin-axis gain difference appears to undergo periodic increases in fluctuation, seemingly corresponding with the warming/cooling cycles observed in the instrument housekeeping sensor temperature values, as shown in Fig. 11. However, both the gain difference and the azimuthal angle difference in the spin plane contribute to the second harmonic of the spin frequency used in the Fourier analysis. None of the calculated absolute angles exhibit this behaviour, which would suggest that the link to the temperature cycling may be coincidental. It is also possible that the phenomenon is noise-related. The data are much cleaner in the tail season, which might lead to reduced fluctuation in the calculation of these parameters, for example. The potential causes cannot be distinguished easily and are beyond the scope of this initial investigation.

In Table 4, the mean value and standard deviations for the elevation angles, theta, have been calculated. The levels of fluctuation in theta are consistent across ranges and spacecraft, with the exception of the spin-axis theta on $\mathrm{C} 1$ for all except range 6 in which the levels are elevated. This might lead to the assumption that the $\mathrm{C} 1$ values are simply consistently elevated. The assumption is borne out when observing 
the theta values for range 2 in all spacecraft as shown in Fig. 12. However, as shown in Fig. 13, an examination of all of the plots reveals spikes of up to $1.5^{\circ}$ occur in ranges 4 and 5 respectively for $\mathrm{C} 1$. This highlights the importance of examining the long-term trends in a number of ways. The variability of the spin-plane thetas has increased later in the mission.

In Table 5, mean value and standard deviations for the azimuthal angles, phi, have been calculated. The levels of fluctuation in phi are consistent across ranges and spacecraft, although initially exceptionally large values were seen in range 5 in $\mathrm{C} 1$ and range 2 on $\mathrm{C} 3$ in the spin axis and range 6 on $\mathrm{C} 3$ in the $y$ coordinate of the spin plane. As shown in the top four plots of Fig. 14, these were caused by spikes in the values for single orbits. The calibration for these orbits was reviewed and corrected, resulting in the improved lower four plots of Fig. 14 and more consistent values in Table 5.

\subsubsection{Individual calibration and instrument housekeeping parameter comparisons}

The calibrated offsets for $\mathrm{C} 2$ and $\mathrm{C} 3$ exhibited cyclical trends in some ranges that merit individual visual comparison with instrument parameters extracted from telemetry. The cyclical trends become more obvious in the latter half of the mission, so range 5 has been chosen as the primary example. On $\mathrm{C} 2, O_{1}$ and $O_{2}$ appear to track the electronics box and outboard sensor temperatures, rising and falling in the same cycle (Fig. 15). $\mathrm{O}_{3}$ shows an inverted trend. On C3, all three offsets appear to track the electronics box and outboard sensor temperatures, rising and falling in the same cycle (Fig. 16).

As mentioned previously, the calibrated spin-plane gains for all spacecraft exhibited cyclical trends in range 2 that merit individual visual comparison with instrument parameters extracted from telemetry. The spin-plane gains for $\mathrm{C} 1$ are the least affected by exceptional single-orbit fluctuations and have thus been chosen for comparison with the electronics box and outboard sensor temperatures as shown in Figs. 17 and 18.

Visual inspection of the plots indicates that there may be a correlation between the warming/cooling sensor temperature cycles and cycling of the spin-plane gain fluctuation. As mentioned above however, it is possible that other factors contribute to the cyclical fluctuation in these calibration parameters, such as the manner in which the gain and azimuthal angle differences are used during the calibration procedure or the noise level in the data. In future, it might be desirable to perform a more thorough data correlation between calibration parameters and temperatures in order to try and discover a temperature coefficient which could be compared with ground data. This was deemed beyond the scope of the present work as an initial survey of parameter comparisons.

\section{Conclusions and future work}

The Cluster mission marks the first time that the magnetometer data from four spacecraft have been calibrated simultaneously in flight. The FGM measurements, and the parameters determined by the FGM post-launch support team for calibrating the outboard magnetometer sensor, span over 11 years. The offsets on $\mathrm{C} 1$ show a steady drift in all ranges (for which there is sufficient data) at the resolution of spacecraft orbits over the course of the Cluster mission to February 2012. The offsets on C2, C3 and C4 remain fairly constant across all ranges. Cyclical trends in the calibration parameters that may be correlated with instrument housekeeping parameters have been identified. Examination of the tabulated means and standard deviations for the gains, elevation and azimuthal angles, has helped to identify cases in which the calibration of certain archived orbits may need to be revisited. However, in general the stability of the outboard sensor calibration parameters over the course of the mission is excellent. Hence, confidence can be placed in the accuracy of the Cluster magnetic field data. In future papers, the features observed in the instrument housekeeping and calibration parameters will be explored further.

Acknowledgements. The Cluster FGM team would like to acknowledge the ESA and the CAA for the ongoing operations and archiving support to fund this work. We acknowledge the STFC for support until UK funding ceased in 2010. We thank the IGEP TU-BS for provision of the FGM data processing software. Finally, we express our regret for the loss of our valuable and respected colleague, Edita Georgescu.

Edited by: V. Korepanov

\section{References}

Auster, H. U., Glassmeier, K. H., Magnes, W., Aydogar, O., Baumjohann, W., Constantinescu, D., Fischer, D., Fornacon, K. H., Georgescu, E., Harvey, P., Hillenmaier, O., Kroth, R., Ludlam, M., Narita, Y., Nakamura, R., Okrafka, K., Plaschke, F., Richter, I., Schwarzl, H., Stoll, B., Valavanoglou, A., and Wiedemann, M.: The THEMIS fluxgate magnetometer, Space Sci. Rev., 141, 235-264, 2008.

Balogh, A., Dunlop, M. W., Cowley, S. W. H., Southwood, D. J., Thomlinson, J. G., Glassmeier, K. H., Musmann, G., Lühr, H., Buchert, S., Acuña, M. H., Fairfield, D. H., Slavin, J. A., Riedler, W., Schwingenschuh, K., and Kivelson, M. G.: The Cluster Magnetic Field Investigation, Space Sci. Rev., 79, 65-91, 1997.

Escoubet, C. P., Russell, C. T., and Schmidt, R. (Eds.): The Cluster and Phoenix Missions, Kluwer Academic Publishers, Dordrecht, 1997.

Brown, P., Carr, C. M., Balogh, A., and Oddy, T. M.: FGM Instrument Users Manual, European Space Agency, 2000. 
Gloag, J. M., Lucek, E. A., Alconcel, L. N., Balogh, A., Brown, P., Carr, C. M., Dunford, C. N., Oddy, T., and Soucek, J.: FGM Data Products in the CAA, Cluster Active Archive: Studying the Earth's space plasma environment, Springer, New York, 2010, 109-128, 2010.

Hedgecock, P. C.: A correlation technique for magnetometer zero level determination, Space Sci. Inst., 1, 83-90, 1975.

Kepko, E. L., Khurana, K. K., Kivelson, M. G., Elphic, R. C., and Russell, C. T.: Accurate Determination of Magnetic Field Gradients from Four Point Vector Measurements - Part I: Use of Natural Constraints on Vector Data Obtained From a Single Spinning Spacecraft, IEEE Transactions on Magnetics, 32, 377-385, 1996, 377-385, 1996
Laakso, H., Taylor, M. G. T., and Escoubet, C. P.: Cluster Active Archive: Studying the Earth's Space Plasma Environment, Springer, New York, 2010.

Walsh, A. P., Forsyth, C., Fazakerley, A. N., Chen, C. H. K., Lucek, E. A., Davies, J. A., Perry, C. H., Walker, S. N., and Balikhin, M. A.: 10 years of the Cluster mission, Astron. Geophys., 51, 33-36, 2010.

Yin, F. and Luehr, H.: Recalibration of the CHAMP satellite magnetic field measurements, Measure. Sci. Technol., 22, 055101, doi:10.1088/0957-0233/22/5/055101, 2011. 